\title{
Outcomes of neonatal screening for hearing loss by otoacoustic emission
}

\author{
Audiology Services, \\ Forest Healthcare, \\ Whipps Cross \\ Hospital, \\ Whipps Cross Road, \\ Leytonstone \\ E11 1NR \\ PM Watkin \\ Dr PM Watkin.

\author{
PM Watkin
}

\begin{abstract}
Aim-To assess universal neonatal screening for bilateral hearing impairments averaging $40 \mathrm{dBHL}$ or worse in the better ear, using transient evoked otoacoustic emission screening (TEOAE) testing.
\end{abstract}

Methods-A three year cohort (14353 infants born from January 1992 to 1995) was screened and subsequently followed up by hearing surveillance methods, including a distraction test screen from 7 months of age. The entire cohort was used to evaluate the outcome of the screen. A subcohort of 8172 district residents was used to evaluate the continuing worth of the distraction test programme.

Results-Nineteen infants (1.3/1000) with a targeted hearing impairment failed the neonatal TEOAE test. Six profoundly deaf infants identified by the TEOAE screen, were fitted with hearing aids at a median age of 16 weeks. One remained without an aid. Of 12 infants with a moderate impairment, only seven accepted hearing aid fitting and the median age of being fitted with an aid increased to 42 weeks. By the time of the analysis 22 children with a targeted hearing impairment $(1.5 / 1000)$ had been identified from the cohort. Of the three missed neonatally, one was cared for elsewhere, another had a progressive loss, and the third had central deafness. Twenty children were ascertained with a congenital peripheral deafness. Of these, eight $(40 \%)$ had risk factors identifiable neonatally. Only the child with central deafness was missed by TEOAE screening and subsequently identified by behavioural tests in infancy. The TEOAE screen outperformed the distraction test in terms of processes and yield and was $25 \%$ less expensive.

Conclusions-The analysis confirmed the worth within the district of the TEOAE hearing screen. It will thus be continued as a universal neonatal screen with the distraction test being retained as a selective screen in the latter half of infancy. (Arch Dis Child 1996;75:F158-F168)

Keywords: TEOAE screen, deafness, distraction test, behavioural tests.

Since 1 January 1992 a universal neonatal hearing screen test-transient evoked otoacoustic emission (TEOAE) has been undertaken in the East London district of Waltham Forest. The practicability of implementng the screen has already been described for a cohort of 14353 infants. ${ }^{1}$ But was the progamme worth implementing?

Early identification and habilitation benefits the hearing impaired child, ${ }^{2-4}$ but measuring real outcomes in terms of the reduction of disability and handicap pose problems, and surrogate indicators are required. ${ }^{5}$ The National Deaf Children's Society (NDCS) ${ }^{6}$ considered that two main outcome measures should be age of confirmation and age at which hearing aids are fitted. Detection of permanent hearing loss (PHL) is worthless without multidisciplinary habilitation, and implementing the screen has implications for other services. The age at which a teacher of the deaf becomes involved with the child and caregivers is a reliable indicator of the availability of the necessary educational services.

Effective introduction of the screen must also reduce the age of identification of different degrees of deafness. This evaluation is ongoing within the district, but requires complete ascertainment and long term follow up of the cohort. Other interim indicators of effectiveness are available. The introduction of a sensitive neonatal screen has implications for existing screens targeting the same impairments. Within the district an acceptably sensitive universal screen undertaken in the latter half of infancy was already in place. ${ }^{78}$ This screen used the Infant Distraction Test (IDT), first described in Manchester over half a century ago. ${ }^{9}$ Because multicentre sensitivity studies had not been undertaken on the TEOAE screen, the IDT was retained. The Third Joint Working Party on Child Health Surveillance ${ }^{10}$ recommends that in this situation the need to continue the IDT should be closely monitored.

Although the screening of all neonates has been recommended in the USA, " selective at risk screening is the model currently in use in the United Kingdom. A selective neonatal screen had previously been used in Waltham Forest, but only $43 \%$ of those with a targeted PHL were identified from a cohort of $10686 .{ }^{12}$ This reduced yield prompted the introduction of the universal screen.

\section{Methods}

Waltham Forest is an East London district with a population of around 220000 . The level of overall deprivation is ranked the 20th highest of all the local authorities in England and Wales. It is home to the largest Pakistani community in London, with one third of the births being to mothers from black and ethnic minority groups. (London Borough of Waltham Forest Policy Analysis Unit, Borough Profile, June 
1996. Tertiary level audiology services are provided from within the district by a unified acute and community service, with the same audiologists providing services to the neighbouring district of Redbridge. Reactive referrals are principally received from general practitioners, parents, ENT departments, paediatric and child health services, with referrals also being made from the child health hearing screening and surveillance programmes. Check lists alerting parents to signs of hearing loss and the health visitor's IDT were both retained throughout the period of evaluation of the neonatal screen.

\section{NEONATAL SCREEN}

The universal neonatal screen has been described in detail. ${ }^{\prime}$ TEOAEs were recorded using the Otodynamics ILO88. ${ }^{13}$ The aim was identification of infants with a congenital bilateral deafness of moderate or worse degree. An initial TEOAE test was undertaken whenever possible before discharge from the maternity unit of the district general hospital (Whipps Cross). Those missing the test in the maternity unit and those born out of district were identified at 4 weeks of age from a Regional Interactive Child Health Computerised System (RICHS) which recorded all district births Those missed in the maternity unit received their initial TEOAE test in the hospital audiology department with entry to the screen being offered up to 3 months of age.

The aims of the screen were explained in a leaflet which was given to all the parents before the TEOAE tests. The results were discussed with the parents by the screeners with the explanation that the aim of the screen was the early identification of those infants with a bilateral hearing impairment sufficient to result in communication disability. All those failing the initial test in both ears required a TEOAE retest. These were undertaken within the audiology department with attempts being made to trace and retest all defaulters when the initial test had been failed in both ears. The parents of those with a unilateral fail were told that intervention was not required neonatally, but that an IDT would be undertaken later in infancy. However, a TEOAE retest was offered if required by the parents. No attempts were made to trace retest defaulters when the initial test had been failed in one ear. Those failing the TEOAE screen were tested by diagnostic ABR (Auditory Brainstem Response) within the audiology department. Usually failure of two sequential TEOAE tests was required before ABR was undertaken, but if there was parental or professional concern TEOAE failure at the initial test was followed by an early appointment for this diagnostic ABR. The TEOAE testing was undertaken by assistant technical officers who were newly employed within the district as neonatal hearing screeners. The ABRs were undertaken by a senior audiologist. Following ABR, the infants were referred for audiological assessment undertaken by the district's senior audiologists. $A$ second ABR was often undertaken at that appointment and the parents were encouraged to observe hearing responses within the home.

If the parents agreed that habilitation was required the infants were assigned to a weekly clinic undertaken jointly with the teacher of the deaf. The intention of this clinic was the provision of seamless care between health service identification and educational service habilitation. The teacher of the deaf undertook home visits on all the infants referred to the joint clinic and no infant was fitted with hearing aids without educational referral.

The screen covered district residents and also non-resident births at Whipps Cross Hospital. It was begun in January 1992, and over the ensuing three years 11606 babies out of a possible 14353 received an initial test. Of those who received this initial test, 2380 (20.5\%) were not Waltham Forest residents. However, 1719 lived in Redbridge and only $661(5.7 \%)$ were from districts where audiological care was provided by other services. The yield from the entire three year cohort was thus used to evaluate the outcome of the screen.

\section{OUTCOME INDICATORS}

The screen aimed to identify those infants with a PHL averaging $40 \mathrm{~dB}$ hearing loss or worse in the better ear. Hearing thresholds measured in other decibel scales were converted to be equivalent to $\mathrm{dB}$ hearing loss. Hearing impairments were classified according to the descriptors recommended by the British Society of Audiology, ${ }^{14}$ but thresholds were averaged at octave intervals from $500 \mathrm{~Hz}$ to $4 \mathrm{kHz}$. The yield included both sensorineural and permanent conductive losses.

Those identified from the neonatal screen (the yield) along with those referred reactively or from subsequent screens (the incremental yield) were ascertained with details of those identified out of district, obtained from educational and health sources. False negative results of the TEOAE screen were thus available. However, the cohort requires long term follow up for identification of all the false negative results, and at this stage the yield cannot be assumed to reflect completely the TEOAE screen sensitivity. The youngest child in the cohort was just over 1 year of age at the time of the evaluation; the eldest was 4 years of age. The present study, therefore, assessed the outcome of the universal screen and the false negative results identified by the time the youngest of the cohort had passed infancy.

Three outcome indicators were used. The age at the $A B R$ was used to indicate the age of confirmation. For those subsequently identified, the age at the diagnostic behavioural test was used. Ages of referral to the educational service and the age of hearing aid fitting were also recorded. Average ages computed the mean, median, and lower and upper quartiles.

Delays in the fitting of hearing aids were evaluated against the NDCS target of an aid being fitted within four weeks of confirmation. ${ }^{6}$ Before habilitation was implemented, the infants were clinically examined and medical 
investigations initiated. This appointment was with the district's senior audiologists. There were full discussions with the parent(s) about the availability of habilitation and the educational home visiting service. This appointment was not always concurrent with the confirmatory ABR, especially if the infant had complex medical needs. It was considered valid that delays in the implementation of habilitation were measured from this clinical appointment. The predominant reasons for further delays were examined from the clinical and educational records. They were categorised as being due to audiological management, avoidable service delay, or family reasons. The latter were often complex but persistent nonattendance, rejection of offered habilitation, and other family instigated delays were grouped together.

The cause of the hearing impairments for all those with a congenital PHL, however identified, were investigated. Aetiologies were also examined to see whether they would have presented neonatally as risk factors for deafness. The criteria used were based on those recommended by the American Joint Committee on Infant Hearing. ${ }^{15}$ Their use within the district has been detailed. ${ }^{12}$

\section{EVALUATION OF HEALTH VISITOR'S DISTRACTION} TEST

A comparative evaluation of the IDT and neonatal screens necessitated the availability of detailed data on individual distraction tests. This was only possible for district residents. Additionally, the coverage of the neonatal screen stabilised only after the end of September 1992. Thus ascertainment of those residents born from 1 October 1992 to 31 December 1994 permitted evaluation of a cohort who had been offered both the neonatal and health visitor screens when both had stable coverage. During this period 8472 district residents were eligible for neonatal screening at birth. An additional 243 moved into the district up to 3 months of age, but 543 had moved out or died before the seven month IDT. The results of the neonatal and IDT screens were evaluated on the $\mathbf{8 1 7 2}$ infants. Those audiologically followed up after the neonatal screen and those referred reactively or from child health surveillance before the IDT were analysed separately.

The mobility of the cohort and the results of the health visitor IDTs were traced through RICHS when each child had reached 1 year of age. Within the district RICHS recorded rates are lower than those available from the health visitors. Thus whenever there was no apparent reason for the IDT having been missed or not completed, clarification and concerns about the hearing were obtained from the health visitor by questionnaire. Seven hundred and six questionnaires were issued with 662 (94\%) returned by the time of the analysis. Results of audiological assessments following failure of the IDT were obtained from audiological records. Comparisons of attendance, failure at each of the two screening stages, and referral for diagnostic assessment were thus available for both screens. Proportions of independent groups were compared using the $\chi^{2}$ test. Differences between means were compared using the $t$ test.

The results of the diagnostic distraction tests undertaken following the two screens were also analysed. A distraction test measuring the threshold of localisation was an important component of the audiological assessment of those failing the health visitor IDT. The same methodology was used in those requiring rehabilitation or follow up after failing the neonatal screen. It was also required for a third group of infants who were referred for audiological assessment before 7 months of age. Comparison of hearing thresholds obtained from the diagnostic IDTs undertaken on the three groups was possible. Thresholds in $\mathrm{dB}$ (A) averaged from 500 to $4000 \mathrm{~Hz}$ were used. The maximum intensity of the hand held warble tone generator in these assessments was $80 \mathrm{~dB}$ and thus those with thresholds $>80 \mathrm{~dB}$ were grouped together. The yield from the two screens was also compared. Those with a moderate or worse PHL identified by the screens were ascertained as were those with otitis media with effusion (OME) which was present at the end of infancy. Referrals for ENT surgery were counted up. Until full pure tone audiometry is available exclusion of all mild PHLs is not possible. However, after exclusion of those with a moderate or worse PHL the mean localisation thresholds in the better ear obtained from the three groups at the diagnostic audiological assessments were compared using the $t$ test.

Cost comparisons were also made. The cost of the neonatal screen has been reported before. ${ }^{1}$ To permit comparison the same costing methodology was used for the health visitor IDT. The neonatal programme was costed to include clerical support, the retesting of initial screen failures, and the undertaking of the threshold ABR examinations. Overheads have been estimated to increase the cost by $40 \%$. Equipment costs amortised over five years and disposables were included. These components were thus also included in the costing of the distraction test, with inclusion of the initial diagnostic evaluation following the screen. Further diagnostic assessments were excluded from both costings. Staff costs were calculated as being midway on the salary scale and included national insurance, superannuation, and London weighting.

\section{Results}

OUTCOME FOR THE COMPLETE COHORT

Age of confirmation

ABR examination was undertaken on 290 out of 337 infants failing the neonatal TEOAE test in both ears. The ABR thresholds in the better ear are detailed in fig 1 .

At the diagnostic audiological examination accompanying or immediately following the $A B R, 23$ infants were considered to have a PHL averaging $40 \mathrm{~dB}$ or worse in the better ear. One infant with a severe hearing loss died at 4 months of age, but the other 22 were 


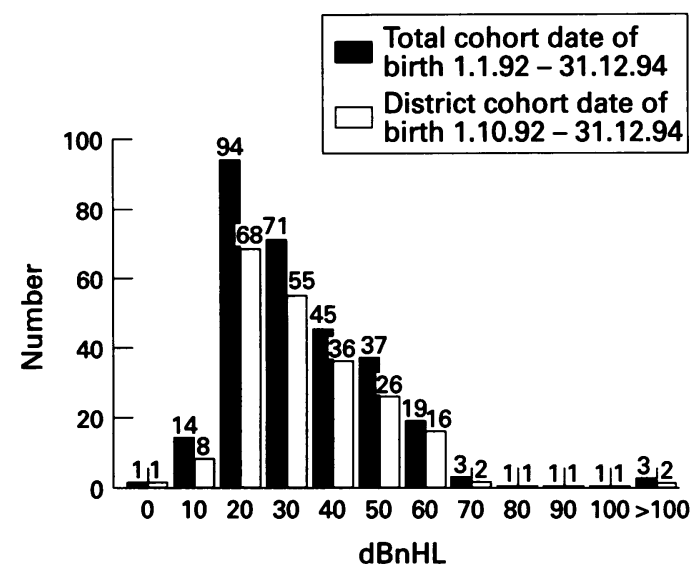

Figure $1 A B R$ threshold in the better ear for the entire cohort and the district subcohort.

followed up with an average of four attendances (range 0-9) after ABR during the remainder of infancy. Eight underwent surgical middle ear ventilation and postoperative $A B R$ during infancy. After resolution of OME or surgical treatment, five of those initially considered to have a moderate impairment had a PHL which turned out to be only mild in degree. The yield of targeted impairments was thus 1.2 per 1000 of the cohort eligible for entry to the neonatal screening programme.

Those subsequently confirmed to have a targeted PHL are detailed in table 1. One child with a moderately severe impairment, associated with bilateral external auditory atresia, was transferred as a neonate to a central London hospital and audiological care was provided outside the district. A profoundly deaf infant who had failed the TEOAE tests brit not attended the ABR was reidentified following the IDT and subsequent audiological assessment. Two other children were also subsequently identified. One had a profound central deafness associated with a neurological and ultimately fatal condition. TEOAEs were present neonatally but complete lack of response to sound was noted when she was 11 months old and ABR confirmed the absence of waveforms at $100 \mathrm{~dB}$. A second child with TEOAEs present shortly after birth had a moderate PHL identified at 2 years 3 months. She had a familial deafness. Many other members of her family have confirmed progressive deafness.

The ages of identification, confirmation, and introduction of habilitation for those with a moderate and severe/profound impairment are detailed in table 2 . The mean age of the initial
TEOAE test was 3.76 weeks in those with a moderate PHL. For those subsequently confirmed with a severe/profound deafness, the mean initial TEOAE test age was just over a week earlier at 2.52 weeks. However, the mean age at which they had an ABR examination of 7.38 weeks was over a month earlier than the age of confirmation of those with a lesser degree of targeted impairment. This relative reduction in age of confirmation for those with a severe or profound deafness was attributable to greater parental and professional concern following TEOAE failure. In four of the six with severe/profound deafness confirmed by $A B R$, this test was undertaken within four weeks of the initial TEOAE failure. In three of the infants there was professional concern and the parents of the other baby were alerted by the TEOAE failure and recognised the presence of deafness within the home. The same level of professional concern was not felt for those with a moderate hearing impairment even though almost the same proportion were identified as being at risk of deafness. The TEOAE test failed to alert the parents to the presence of a PHL requiring habilitation in any of the 12 with this degree of deafness, and in only one was the screening process accelerated so that $A B R$ was under one month from the initial TEOAE failure.

\section{Referral to educational services}

Of the total of eight children with a severe or profound deafness, only six were referred to the educational service for habilitation. The wishes of the parents of the child with the fatal neurological condition were respected with no referral being made. The profoundly deaf child, who failed to attend the neonatal ABR but who was reidentified following the IDT, once again failed to attend all appointments after the diagnostic behavioural assessment. The six who were identified after the neonatal screen were referred at a mean age of $\mathbf{1 5 . 2}$ weeks (SD 14.2 weeks) with a median age of 11.4 weeks (table 2). The cumulative distribution of age of educational referral for the 6 is detailed in fig $2 \mathrm{~A}$.

Thirteen of the 14 children with a moderate PHL were referred to a teacher of the deaf. The parents of a child with Down's syndrome whose hearing impairment was identified neonatally, did not wish to be referred and moved out when the child was 18 months old. The child cared for out of district was referred at 37 weeks and the child with the familial progres-

Table 1 Yield of children with a confirmed permanent hearing loss*

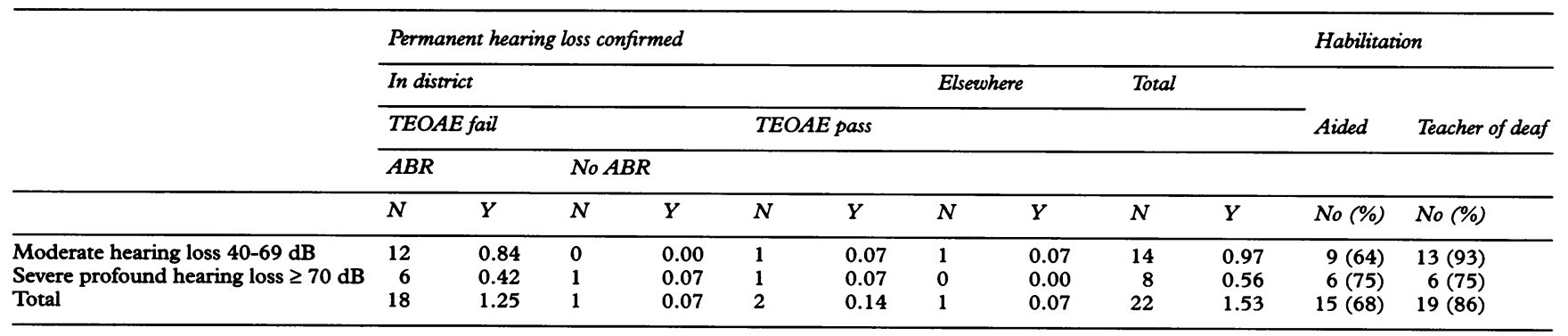

$\star N=$; Y Yield/1000 form cohort of $14353 ; \%=$ percentage of total identified. 

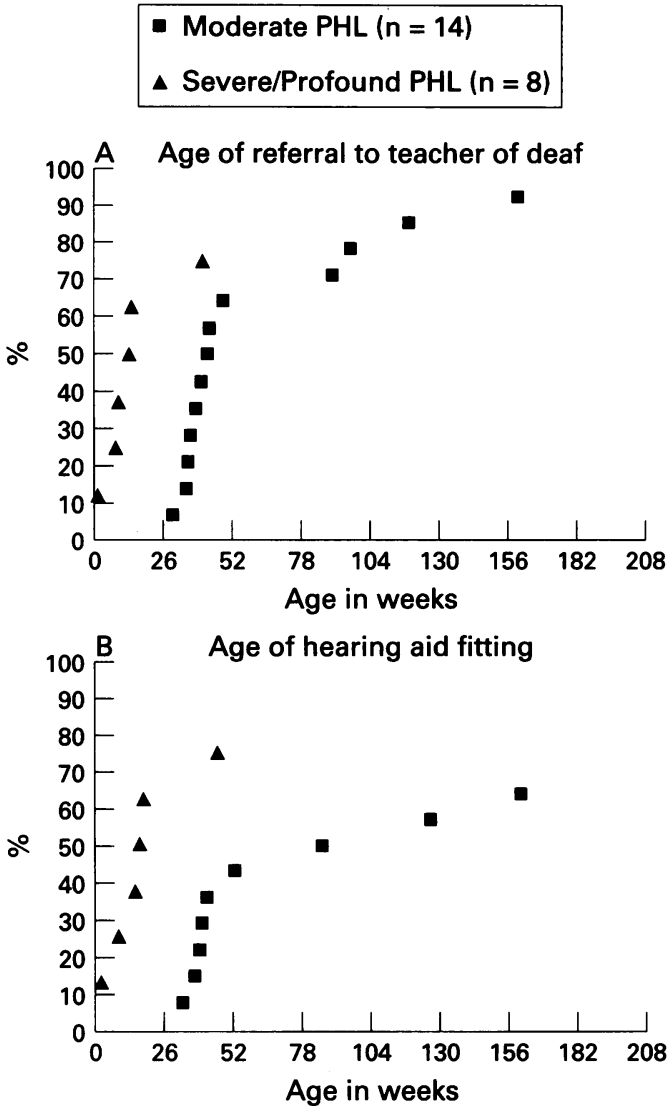

Figure 2 Cumulative distribution of age of referral to the teacher of the deaf and the age of hearing aid fitting

sive loss was referred just after her third birthday. The mean age of referral of the 11 identified by the neonatal screen was 56.7 weeks (SD 30.2 weeks). However, the median age was 43.6 weeks (table 2) with eight of the 11 being referred in infancy. The cumulative distribution of age of educational referral for the 13 is detailed in fig $2 \mathrm{~A}$.

Age of hearing aid fitting

Of the eight children identified with a severe or profound deafness, only the six identified by the neonatal screen were fitted with hearing aids. Neither the child with the central

Table 2 Ages of identification, confirmation, and rehabilitation

\begin{tabular}{|c|c|c|c|c|c|c|}
\hline & \multirow[b]{2}{*}{ No } & \multicolumn{5}{|c|}{ Age in weeks } \\
\hline & & Mean & $(S D)$ & $25 \%$ & $50 \%$ & $75 \%$ \\
\hline \multicolumn{7}{|l|}{$\begin{array}{l}\text { Moderate impairment 40-69 dBHL } \\
\text { (total identified = 14): }\end{array}$} \\
\hline Failed initial TEOAE test & 12 & 3.76 & $(5.75)$ & 0.13 & 0.31 & 7.81 \\
\hline ABR examination & 12 & 12.1 & $(4.10)$ & 9.47 & 12.9 & 16.9 \\
\hline Referred to teacher of deaf & 11 & 56.7 & $(30.2)$ & 36.9 & 43.6 & 90.0 \\
\hline Fitted with hearing aids & 7 & 59.1 & (34.3) & 36.9 & 41.9 & 85.0 \\
\hline Hearing loss confirmed elsewhere & 1 & 16.0 & & & & \\
\hline Referred to teacher of deaf & 1 & 37.0 & & & & \\
\hline Fitted with hearing aids & 1 & 40.0 & & & & \\
\hline Hearing loss confirmed by later tests & 1 & 136.2 & & & & \\
\hline Referred to teacher of deaf & 1 & 159.7 & & & & \\
\hline Fitted with hearing aids & 1 & 159.7 & & & & \\
\hline \multicolumn{7}{|c|}{$\begin{array}{l}\text { Severe/profound impairment } \geq 70 \mathrm{dBHL} \\
\quad \text { (total identified }=8 \text { ): }\end{array}$} \\
\hline Failed initial TEOAE test & 7 & 2.52 & $(2.86)$ & 0.11 & 1.04 & 5.91 \\
\hline ABR examination & 6 & 7.38 & (3.57) & 3.53 & 9.22 & 9.78 \\
\hline Referred to teacher of deaf & 6 & 15.2 & (14.2) & 7.18 & 11.4 & 22.2 \\
\hline Fitted with hearing aids & 6 & 17.9 & $(15.3)$ & 7.18 & 15.9 & 25.1 \\
\hline Hearing loss confirmed elsewhere & 0 & & & & & \\
\hline Hearing loss confirmed by later tests & 2 & 60.6 & $(7.10)$ & & & \\
\hline Referred to teacher of deaf & 0 & & & & & \\
\hline Fitted with hearing aids & 0 & & & & & \\
\hline
\end{tabular}

neurological deafness nor the child whose parents persistently failed to attend were so fitted. The mean age of hearing aid fitting was 17.9 weeks (SD 15.3 weeks) with the median age being 15.9 weeks (table 2).

Of the 12 children with moderate impairments identified by the neonatal screen, seven had been fitted with aids by the time of the analysis. The mean age was 59.1 weeks with a median age of 41.9 weeks (table 2). The child cared for out of district was fitted with a hearing aid at 40 weeks, and the child with progressive deafness at 160 weeks. The cumulative distribution for all aid fittings is shown in fig 2B.

\section{Delays in fitting of aids}

The delays in aiding were investigated for those identified by the neonatal screen. The mean delay from the age of the clinical appointment accompanying or immediately following the final confirmatory $\mathrm{ABR}$ for the seven with a moderate imapirment was 43.5 weeks (SD 34.8 weeks). The median delay was 29.1 weeks with none meeting the NDCS target of an aid being fitted within four weeks of confirmation. Five confirmed by the neonatal screen were not fitted with an aid. They had a mean age of 2.5 years (SD 0.8 years) at the time of the analysis. The youngest was 18 months old with the eldest being 3.3 years. All of these children who had not been fitted with aids retained moderate impairments for family reasons. In the seven who had been fitted with an aid, initial audiological uncertainties resulted in habilitation being delayed until the second half of infancy. In five of them both parents and professionals had accepted the need to introduce hearing aids in infancy. In the other two, after initial audiological uncertainty had been resolved, family reasons, including aid refusal, delayed corrective measures. Thus of the 12 with a moderate impairment identified by the neonatal screen, only five were fitted with an aid in infancy.

The situation was different for those identified with either a severe or profound impairment. The mean delay in fitting an aid after the clinical appointment accompanying or immediately following the final confirmatory $A B R$, for the six with a severe or profound impairment, was 6.3 weeks (SD 12 weeks). However, one child had auditory habilitation delayed by 31 weeks following a confirmatory ABR at under 4 months. Fitting of a hearing aid was not accepted by the family until behavioural responses made clear the degree of the infant's loss. The remaining five all met the NDCS target of receiving an aid within four weeks of confirmation. Of the two children with profound deafness who were were not fitted with an aid, one with central deafness died at 26 months. Unfortunately, the other remained without an aid for family reasons. He was 25 months old at the time of the analysis.

Although mild and unilateral PHLs were not targeted for identification, 13 infants were identified from the neonatal screen with a mild impairment and five with a permanent unilateral loss. Of those with a mild PHL, five were 
Table 3 Presence of risk factors in those with a targeted congenital sensorineural or permanent conductive hearing loss

\begin{tabular}{lcll}
\hline Aetiology & No & Admitted SCBU & Neonatal risk factor \\
\hline Unknown & 7 & 1 & 1 \\
Familial & 5 & 0 & 1 \\
$\quad$ Clinically undifferentiated & 1 & 1 & 1 \\
$\quad$ Clinically differentiated & 0 & 0 & 0 \\
Congenital infection & & & 1 \\
Malformation or syndrome & 1 & 1 & 1 \\
$\quad$ Down's syndrome & 1 & 1 & 1 \\
$\quad$ Treacher-Collins syndrome & 1 & 1 & 0 \\
$\quad$ Ring chromosome 22 & 1 & 0 & 0 \\
$\quad$ Mucopolysaccharidosis & 1 & 0 & 1 \\
$\quad$ Skeletal dysplasia & 1 & 1 & 8 \\
$\quad$ Schwachman and ear canal atresia & 1 & 1 & 1 \\
Total & 20 & 7 & \\
\hline
\end{tabular}

SCBU: special care baby unit

Table 4 Comparison of TEOAE and IDT processes

\begin{tabular}{|c|c|c|c|c|}
\hline & \multicolumn{2}{|c|}{$T E O A E$ tests } & \multicolumn{2}{|c|}{ Health visitor's distraction tests } \\
\hline & No & (\%) of cohort & No & (\%) of cohort \\
\hline Eligible for test & 8172 & $(100)$ & 7777 & $(100)$ \\
\hline Initial screening test undertaken & 7593 & (92.9) & 6763 & $(86.9)$ \\
\hline Failed initial test & 1031 & (12.6) & 1520 & (19.5) \\
\hline $\begin{array}{l}\text { Referred immediately for } \\
\text { diagnostic test }\end{array}$ & 101 & $(1.2)$ & 51 & $(0.7)$ \\
\hline Screen retest required & 930 & (11.4) & 1469 & (18.9) \\
\hline Retest undertaken & 823 & $(10.1)$ & 1318 & $(16.9)$ \\
\hline Failed retest & 141 & $(1.7)$ & 611 & $(7.8)$ \\
\hline Total requiring diagnostic test & 242 & $(2.9)$ & 662 & $(8.5)$ \\
\hline Diagnostic test undertaken & 217 & $(2.6)$ & 521 & $(6.7)$ \\
\hline Total not completing programme & 711 & (8.7) & 1306 & $(16.8)$ \\
\hline
\end{tabular}

initially considered to have a moderate degree of hearing impairment following the neonatal ABR. An additional child with a high frequency impairment averaging within the mild range was missed by the TEOAE test. She is the only one to have been fitted with hearing aids, although eight of the others have been notified to the educational services. The five identified with unilateral impairments remained under audiological review but had not been notified to the educational services nor fitted with an aid.

\section{Risk factors for deafness}

The cause of the deafness was investigated in the 20 children with a congenital targeted peripheral impairment. Aetiologies were identified in $13(65 \%)$ and are detailed in table 3. However, they were not always apparent at birth. Two of the six families with a dominantly inherited hearing impairment failed to disclose its presence within a first degree relative when directly questioned by the audiologist at the ABR examination. One of the siblings to whom this applied wore hearing aids, and another mother with a clinically obvious hearing loss declined her own testing. Familial deafness was

Table 5 Infants referred to audiology department when aged less than 7 months

\begin{tabular}{lr}
\hline Reason for referral & No \\
\hline Risk factor for deafness present but TEOAE screen passed & 44 \\
TEOAE screen failed in one ear, and IDT follow up required & 75 \\
TEOAE failed unilaterally and ABR consistent with unilateral permanent hearing loss & 8 \\
TEOAE screen not completed but IDT follow up required & 20 \\
TEOAE screen failed bilaterally & 242 \\
Reactive referral with TEOAE passed in at least one ear & 6 \\
Total & 395 \\
\hline
\end{tabular}

noted in another two only after hearing tests had been undertaken on both parents. Six of the children had a named syndrome or chromosomal abnormality, but in only four was clinical diagnosis reached within three months of birth. One had a mucopolysaccharidosis identified late in infancy and another an unnamed skeletal dysplasia not associated with craniofacial dysmorphology and not recognised to be associated with hearing loss. One child with a severe loss had had severe birth asphyxia and was identifiable through admission to the special care baby unit and the known perinatal risk factor. Another child without other risk factors was identifiable through admission to the unit but he was only four weeks premature and had no perinatal illness. Eight of the $20(40 \%)$ were identified as at risk within three months of birth, with five of the $13(38.5 \%)$ with a moderate impairment that was identified and three of the seven $(43 \%)$ severely or profoundly deaf having neonatal risk factors (one birth asphyxia; one ring chromosome; one Treacher-Collins syndrome).

\section{Evaluation of the Infant Distraction Test}

In total 8172 infants were eligible for both the neonatal TEOAE and infant distraction tests. The initial TEOAE test was undertaken on 7593 (92.9\%) neonates. By 7 months of age 395 infants were already under the care of the audiology service and thus 7777 infants eligible for neonatal testing should have received the health visitor's IDT screen. It was undertaken on $6763(86.9 \%)$.

The results of the TEOAE and IDT screens are detailed in table 4 . The absence of an emission in both ears constituted a TEOAE screen failure. The ABR results in the better ear for the 217 infants tested because of bilateral TEOAE failure, are given in fig 1 .

By 7 months of age 395 infants were already under audiological care for reasons summarised in table 5. Those 242 failing the neonatal screen bilaterally were mandatory audiology follow ups. An additional 153 infants were being followed up during the first half of infancy. The six who were referred reactively because of professional or parental concern had passed the neonatal screen in at least one ear. Most ( 83 of the 153) were reviewed on parental request because they had failed the TEOAE test unilaterally. Forty four who passed the TEOAE test had risk factors which may have resulted in a progressive hearing loss and they remained under review. Included were infants with a family history of deafness, neurodevelopmental disorders, syndromes, dysmorphologies and cleft palates.

\section{Comparison of coverage}

Defaulters of the initial and repeat tests, along with screen failures not attending for diagnostic assessment or ABR, were considered not to have been screened for calculation of coverage. In total 1014 infants failed to receive the initial health visitor test, with a further 292 failing to complete the programme. In comparison 579 neonates did not receive an initial TEOAE test, 
Table 6 Mean localisation thresholds obtained at audiological assessments excluding those identified with permanent hearing loss

\begin{tabular}{lllll}
\hline & & \multicolumn{2}{l}{ Threshold in better ear in dBA } & \\
\cline { 3 - 4 } Source of referral & No & Mean & (SD) & t test \\
\hline TEOAE screen & 203 & 41.2 & $(7.5)$ & $1 \mathrm{v} 2 \mathrm{t}=1.67$ \\
Health visitor's distraction test & 520 & 40.2 & $(6.5)$ & $2 \mathrm{v} 3 \mathrm{t}=1.29$ \\
Audiological referral < 7 months & 121 & 41.1 & $(7.0)$ & $1 \mathrm{v} 3 \mathrm{t}=0.12$
\end{tabular}

with a further 132 not completing the programme. The coverage of the neonatal screen was $91.3 \%$ with the health visitor's IDT coverage being $83.2 \%$.

The reasons for non-attendance at the IDT were investigated from the health visitors' questionnaires. Of the 1306 infants who failed to complete the programme, the reasons were clear for 600 . Seven hundred and six questionnaires were sent to the health visitors. Forty four were unanswered, and for the purposes of analysis these were categorised as having no recorded reason for failing to complete the IDT programme. Of the total of 1306 who failed to complete the programme, $582(45 \%)$ were called on at least two occasions, but they failed to attend. A further 331 (25\%) failed to attend but were not resident in one place. Forty one had moved in before their child reached the age of 4 months. This represented $17 \%$ of the 243 moving in and this coverage was not significantly lower than that for the remainder of the eligible cohort $\left(\chi^{2} 0.001\right.$, df $\left.1, P>0.5\right)$. However, of the 395 infants who moved out of the district between 7 months and 1 year of age, $290(73 \%)$ had not completed an IDT. There was no recorded reason for the nontesting of the remaining $393(30 \%)$ infants.

The reasons for non-attendance for the neonatal screen were not investigated in the present study, but of 243 moving in before four months, only $73(30 \%)$ received a TEOAE test.

Audiological asssessments

Of the 242 failing the neonatal screen, 214 $(88 \%)$ were followed up for a diagnostic IDT. Of the 153 under review before the IDT, 133 $(87 \%)$ were followed up to the end of infancy. This group contained those infants with neurodevelopmental problems, and although $122(80 \%)$ were successfully tested to threshold, by 1 year of age 11 had not reliably been tested by the IDT. These 11 were all followed up and none had a PHL. However, because they were not testable by the IDT, they were omitted from the threshold comparisons. Of the 662 failing the health visitor's IDT, 521 $(79 \%)$ attended a diagnostic assessment. The comparison of localisation thresholds obtained from the three groups is graphically detailed in fig 3. The mean thresholds, excluding those infants with a PHL, were compared (table 6). There was no significant difference $(P>0.05)$ among the three groups.

Comparison of the yields

The yield present at the end of infancy is detailed in table 7 . From the neonatal screen
11 infants were identified with a PHL of $>40$ $\mathrm{dB}$ in the better ear, and 112 were identified with OME. Of those identified with OME, 35 (31\%) were referred for surgical middle ear ventilation. A larger number of infants $(n=304)$ with $O M E$ were identified from the health visitor's IDT. However, only $15(5 \%)$ had been referred for ENT surgery by the end of infancy. The previously detailed infant who had failed the neonatal screen in both ears but who had failed to reattend was the only child with a targeted peripheral PHL identified by the health visitor's IDT. From the 153 referred before the IDT, 64 had OME present at the distraction test and $12(19 \%)$ were referred for surgical middle ear ventilation. The child previously detailed with central deafness and a degenerative neurological disease was the only infant identified from this group with a PHL.

From this subcohort there was a total yield of 1.6/1000 infants with a PHL, with an incremental yield of $0.1 / 1000$ from the health visitor's IDT. Twelve of the 13 infants with a PHL had failed the TEOAE test. Although of
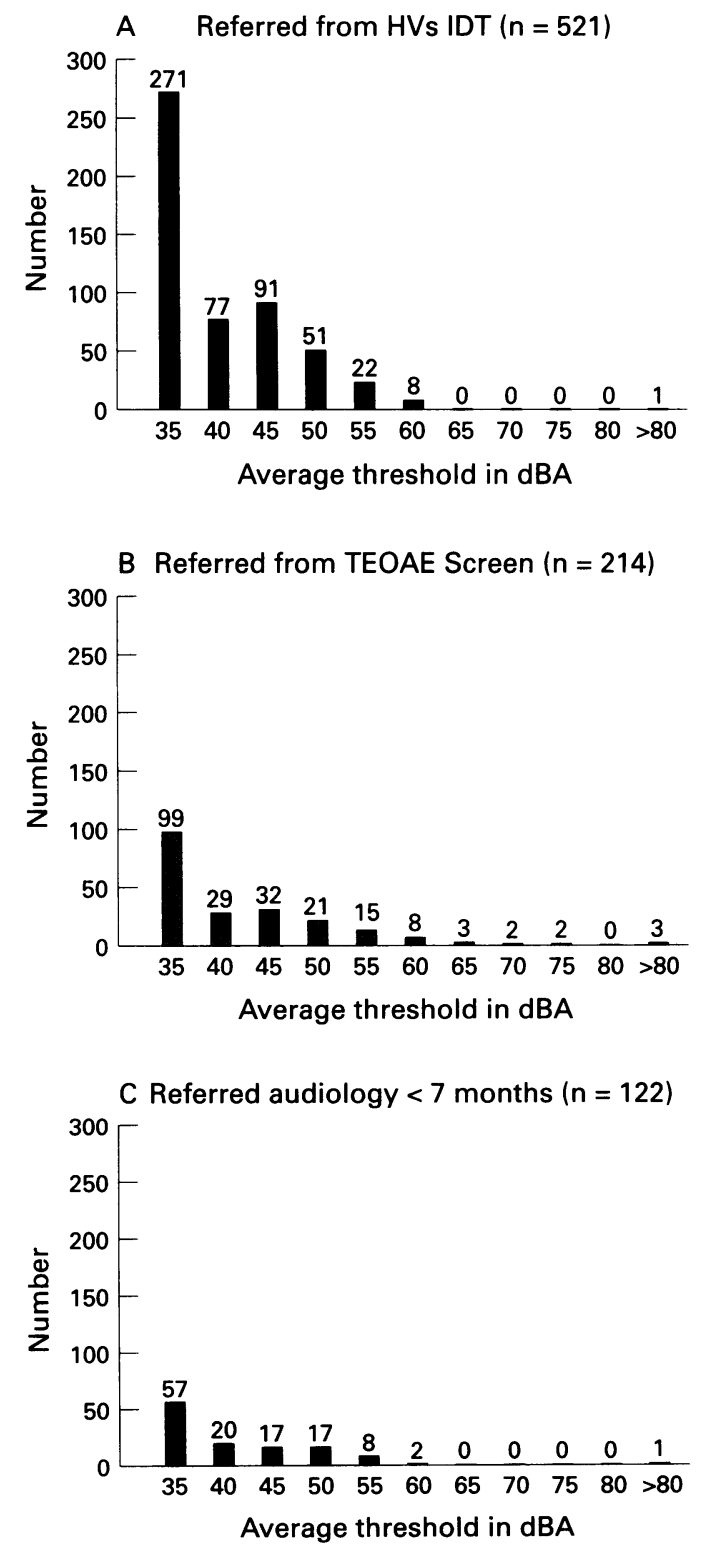

Figure 3 Hearing thresholds in the better ear obtained by the distraction test 


\begin{tabular}{|c|c|c|c|c|c|c|c|c|c|}
\hline & \multicolumn{3}{|c|}{ Failed TEOAE screen } & \multicolumn{3}{|c|}{$\begin{array}{l}\text { Failed health visitor's } \\
\text { distraction test }\end{array}$} & \multicolumn{3}{|c|}{$\begin{array}{l}\text { Audiological referral }<7 \\
\text { months }\end{array}$} \\
\hline & $N$ & $(\%)$ & $Y$ & $N$ & (\%) & $Y$ & $N$ & $(\%)$ & $Y$ \\
\hline $\begin{array}{l}\text { Targeted permanent hearing loss } \\
\text { Otitis media } \\
\text { ENT consultation }\end{array}$ & $\begin{array}{r}11 \\
112 \\
35\end{array}$ & $\begin{array}{l}(4.5) \\
(52) \\
(14)\end{array}$ & $\begin{array}{r}1.3 \\
13.7 \\
4.3\end{array}$ & $\begin{array}{r}1 \\
304 \\
15\end{array}$ & $\begin{array}{l}(0.2) \\
(58) \\
(3)\end{array}$ & $\begin{array}{r}0.1 \\
37.2 \\
1.8\end{array}$ & $\begin{array}{l}1 \\
64 \\
12\end{array}$ & $\begin{array}{l}(0.8) \\
(52) \\
(10)\end{array}$ & $\begin{array}{l}0.1 \\
7.8 \\
1.5\end{array}$ \\
\hline
\end{tabular}

$\mathrm{N}=$ number of infants; $\%=$ percentage of those receiving a diagnostic distraction test; $\mathrm{Y}=$ yield/1000 from total cohort of 8172 .

the total yield of 59/1000 infants with OME, 37 were identified by the health visitor's IDT, the proportion with $\mathrm{OME}$ identified from each of the three groups was not significantly different $\left(\chi^{2} 0.840\right.$, df $\left.2, P>0.50\right)$. Of the 521 infants who failed the health visitor's IDT and also received a diagnostic assessment, 305 had either OME or a targeted PHL. The false positive rate (FPR) was $41.5 \%$, with a positive predictive value (PPV) of $58.5 \%$. Of the 214 infants who received a diagnostic IDT following bilateral failure of the neonatal screen, 123 had either OME or a targeted PHL. The FPR was thus $42.5 \%$ with a PPV of $57.5 \%$.

\section{Comparative costs}

The cost of the health visitor's IDT by year is detailed in table 8 . With a $20 \%$ nonattendance rate, and the requirement to reappoint an additional $20 \%$ who failed the first screening test, a total of just under 6000 appointments were required annually to obtain the achieved IDT coverage. With each test requiring 15 minutes, 1500 health visitor and clinic aid hours were required over the year. This was equivalent to 1.0 whole time equivalent of each, allowing for one session of administration a week. The initial audiological assessments required for the $8.5 \%$ failing the IDT screen was undertaken by a senior audiologist working with an assistant technical officer. With a slightly higher non-attendance rate of $25 \%$, one session a week was required for these assessments with nine infants appointed to each session. The equipment costs for the screen included the test items of a Manchester Rattle, and a C $(512 \mathrm{~Hz})$ and $\mathrm{G}$ $(1600 \mathrm{~Hz})$ chime bar for all the health visitors, and a warble tone generator and admittance machine for use by the audiologists. The total annual cost of the IDT screen was marginally higher than the cost of the TEOAE screen. However, $20 \%$ of those tested by the neonatal screen were non-district residents. Thus after including a sum of $40 \%$ for overheads and

Table 8 Costs of providing screens

\begin{tabular}{lll}
\hline & \multicolumn{1}{l}{ Cost $(\ell)$} & \\
\cline { 2 - 3 } & TEOAE screen & Health visitor's distraction test \\
\hline Equipment (5 year amortisation) & 6680 & 820 \\
$\begin{array}{l}\text { Disposables: } \\
\text { Stationery, postage, probes, etc }\end{array}$ & 3100 & 1000 \\
Staff: & N/A & 1.0 WTE 23636 \\
$\quad$ Health visitor & N/A & 1.0 WTE 11420 \\
Clinic aid & 0.2 WTE 6200 & 0.1 WTE 3100 \\
Senior audiologist & 1.9 WTE 22603 & 0.1 WTE 1053 \\
Assistant technical officer & 0.5 WTE 5635 & 0.5 WTE 5635 \\
Clerical assistant & 44218 & 46644 \\
Total cost/year & 61905 & 65301 \\
Total cost $+40 \%$ overhead & 13.75 & 18.14 \\
Cost/child screened & & \\
\hline
\end{tabular}

reducing the cost of the neonatal screen by $20 \%$, the cost of each IDT screen at just over $£ 18$ was $25 \%$ more expensive than the cost of each neonatal screen.

\section{Discussion}

A previous report ${ }^{1}$ on the cohort of 14353 born from January 1992 to January 1995 showed that it had been possible to implement a practicable universal neonatal screen using TEOAE tests followed by diagnostic ABR. The value both to the district and those targeted for identification requires long term evaluation. The youngest child neonatally tested will be 5 years old at the turn of the century. However, some important measures of screen worth, and the implications of introducing the screen, have been investigated in the interim.

Haggard ${ }^{16}$ considered that the main objective of early infant screening should be to detect severe and profound prelingual deafness. Sancho et al ${ }^{17}$ noted that a case already exists for the early fitting of hearing aids to children with moderate degrees of deafness. The 1993 National Institutes of Health Consensus Statement ${ }^{11}$ also recommended the detection of neonates with moderate and worse hearing impairment. Kennedy et al ${ }^{18}$ in their preliminary report of universal neonatal TEOAE screening also defined substantial hearing impairment as moderate or worse in the better ear. However, outcomes differ for these different degrees of impairment.

From the cohort of 14353 infants, 21 children were identified with a congenital PHL averaging $40 \mathrm{~dB}$ hearing loss or worse in the better ear. This yield of 1.46 per 1000 was not entirely from the neonatal screen, but apart from one child managed out of district and another with central deafness, all had failed the TEOAE tests. TEOAE screening detects cochlear dysfunction and will inevitably be insensitive to central deafness. However, the benefits of identifying such losses are unclear. Twice as many infants were identified with moderate impairments as were severely or profoundly deaf.

Those with a severe or profound deafness had their hearing impairment confirmed at a median age of 9 weeks. Following the neonatal tests the presence of deafness was evident to both the audiologists, and with a single exception, to the parents. Behavioural observation of the infants' responses to sound allowed for early acceptance of the impairment with involvement of the teacher of the deaf at a median age of 11 weeks and a hearing aid fitting at 16 weeks. Five of the six children fitted with an aid were referred to the educational services by 6 months of age, and this allowed 
the NDCS targets for the implementation of multidisciplinary habilitation to be met in all but one of the infants.

The ABR confirmation of moderate impairments was at a median age of 13 weeks. Although the initial TEOAE test was only a week later than that undertaken on those with a more severe loss, there was not the same level of parental or professional concern following the initial TEOAE failure and consequently the confirmatory ABR was relatively delayed. Even after the ABR there were delays in recognising the requirement to implement habilitation. One hundred and one of the cohort who failed the TEOAE tests bilaterally had ABR thresholds from 40 to $60 \mathrm{~dB}$ hearing loss, and in those with a moderate PHL there initially remained audiological uncertainty that the loss was entirely sensorineural. In five of 17 infants considered to have a moderate PHL immediately following the $\mathrm{ABR}$, the permanent component of the loss eventually turned out to be only mild. In the 12 with a confirmed moderate PHL it was difficult to be sure early after the ABR that the hearing loss was indeed permanent and that habilitation and fitting with an aid were required. Certainty grew during the second half of infancy. This allowed eight out of the 12 to be referred to the teacher of the deaf during the second half of infancy, with five also being aided during this period. However, continuing uncertainty was experienced by some of the parents and three children who had their impairment confirmed during the first half of infancy were referred for rehabilitation when they were around 2 years of age. Of these, only two were fitted with an aid. Five children with permanent hearing impairments of moderate degree (all aged over 18 months at the time of the analysis) remained without an aid. The median age of fitting was delayed to 42 weeks, with referral to the educational services by 44 weeks. The NDCS target of fitting an aid within a month of confirmation was not achieved.

Such uncertainties are not surprising. Moderate hearing impairment presents very subtly. Behavioural responses to sound are difficult to evaluate even for the most experienced observers, ${ }^{19}$ with automated neonatal tests based on such responses relatively insensitive to those with moderate losses. ${ }^{20}$ Reducing the age of audiological certainty can be envisaged with the more effective use of appropriate diagnostic procedures. Bone conduction ABR ${ }^{22}{ }^{23}$ and oto-admittance testing using high frequency probe tones ${ }^{24}$ will help establish which of those with moderate impairments on air conduction $A B R$ actually have a $P H L$ requiring early intervention. But improvements in diagnostic acumen alone will not enable appropriate habilitation of those with more moderate impairments. Quite reasonably, many parents are reluctant to allow referral to a teacher of the deaf and the fitting of hearing aids to their infant unless they notice a problem-yet the aim of the screen is to intervene before disability becomes evident.

Whether habilitation in the first half of infancy is beneficial for children with moderate hearing impairments has not yet been firmly established. Before the introduction of neonatal screens such children were identified within the reported district at a mean age of 18 months. ${ }^{8}$ The IDT was relatively insensitive to this degree of impairment, with a screen test sensitivity of $36 \%$ being recorded in the early 1980s. Identification at 18 months often delayed the implementation of consistent habilitation until the children were approaching 3 years of age. Identification in early infancy, when accompanied by parental acceptance, permits consistent hearing aid use to be established in infancy. Clinically, this seems to be worth while for their communication skills and overall development, but the benefits of early intervention have been demonstrated on those with congenital severe or profound deafness ${ }^{24}$ and controlled evaluations of early intervention for lesser degrees of impairment are still required. Ramkalawan and Davis concluded from their study on the effects of hearing loss and the age of intervention on speech, ${ }^{3}$ that detrimental effects do result from delayed intervention even in children with milder hearing losses. If intervention in infancy is of scientifically proved benefit, then the current outcome evaluation shows that diagnostic and habilitative strategies for those with moderate losses require further development.

Robinshaw reported case studies of congenitally profoundly deaf infants given hearing aids between 3 and 6 months of age, ${ }^{4}$ and concluded that variables in addition to auditory stimulation should be examined and different programmes of (re)habilitation explored. The present evaluation would entirely support these conclusions for those children with moderate degrees of impairment. Many of those with a PHL can be identified by neonatal hearing screening, but for those with less severe impairments, methods of habilitation implemented in infancy which only promote the use of amplification are rejected. The further investigation of methods which would allow parents to confirm the presence of less severe impairments after the clinical tests would increase the worth of detecting them.

Sancho et al ${ }^{17}$ perceptively noted that extending the range of losses that are detected has important implications for the habilitation services. These implications are clear from the cumulative distribution of age of referral to the educational service. Identification is worthless without the availability of adequate (re)habilitation programmes.

The need to implement a neonatal screen universally within the district was also investigated. Selective at risk screening is cost effective and may identify up to $70 \%$ of the hearing impaired neonates. ${ }^{25}$ However, within the reported district it had only identified $43 \%$ of targeted impairments. ${ }^{12}$ The present analysis confirmed the difficulties. Retrospective examination of the aetiologies suggested that 13 of the $20(65 \%)$ with congenital peripheral impairments were at risk. However, fewer would have been thus identified neonatally. Although just under a third had a familial deafness, only one third of them would have been identified by simple enquiry. The same 
proportion had a hearing impairment associated with a congenital malformation or syndrome. However, one third of these conditions were not identified at an age which would have facilitated referral in the first half of infancy. Altogether, 865 infants were admitted to the special care baby unit and seven had a targeted impairment. This yield of 1 in 124 has been reported before. ${ }^{126}$ Only one infant had a deafness acquired through perinatal illness, and this child died in infancy. However, testing every admission to the unit would have identified seven out of the 20 (35\%) targeted impairments. This would have increased to eight out of $20(40 \%)$ if admitted family histories had been screened, and to 10 out of $20(50 \%)$ if all known family histories had been identified. These results confirmed those previously found in the district and the place of the universal screen.

What, then, was the continued place of the health visitor's IDT? This had been successfully implemented within Waltham Forest for over quarter of a century. It was feared that with the introduction of the TEOAE screen it would be abandoned by both parents and professionals. Measures of the coverage show that this was not the case. The proportion of eligible infants receiving an initial IDT in the district has remained at around $86 \%$ for many years. ${ }^{7-12}$ The introduction of the neonatal screen did not reduce this. However, the Third Joint Working Party on Child Health Surveillance ${ }^{10}$ suggested that when a sensitive universal neonatal screen is introduced, continuation of the health visitor's IDT cannot remain cost effective. The worth of continuing this later screen was evaluated. The specificity of the neonatal screen during the three years reported was low and has subsequently been improved. The measures of the neonatal screen processes thus present a worse case scenario. Despite this, on all measures including proportions receiving and failing the initial test, proportions requiring and failing the retest, the number requiring referral for assessment, and the overall coverage- the neonatal screen outperformed the IDT. Yet the neonatal screen was $25 \%$ cheaper to implement than the IDT.

Comparison of yields obtained from the screens was biased because the IDT was used on the subcohort already screened neonatally. If both PHL and hearing loss due to OME were considered a desirable yield, the validity of both screens in terms of the PPV was very similar. The main difference was in the type of yield. Of the 13 infants from this subcohort, detected with a PHL, 12 had failed the TEOAE tests. Admittedly, one had failed the neonatal screen but had been lost to follow up, but his reidentification by the IDT did not, unfortunately, permit his habilitation. Only the infant with central deafness was a false negative of the neonatal screen. There was thus no incremental yield of children with a PHL who benefited from detection by the IDT screen.

The yield of infants with OME was greater from the IDT. Fewer of these children were referred for ENT management. However, this statistic should be considered with caution.
Persistence criteria dictate ENT referral and would be greater in those where OME had been present from early infancy. Only long term follow up of those identified by the IDT will allow for a true comparison of referral rates. It has been suggested that TEOAE screen failure may predict future middle ear dysfunction. ${ }^{18}$ Howie in $1975^{27}$ also suggested that age of onset of middle ear disease predicts the requirement for later intervention. However, after exclusion of those with a targeted PHL, the average hearing threshold at the IDT of those who had failed the neonatal screen was no worse than the hearing level of those who had passed the TEOAE screen, but subsequently failed the health visitor's IDT programme. The proportion with $\mathrm{OME}$ was also the same.

The study confirmed that with the implementation of the universal neonatal screen, the main function of the IDT within the district became the identification of OME. This problem was predicted by the Joint Working Party on Child Health Surveillance. ${ }^{10}$ This report considered that "... OME discovered by screening ... is not generally regarded as the function of the distraction test ..." Questionnaires sent to the health visitors in the early days of the screen asked whether they thought it worthwhile and whether they still considered that the IDT should be undertaken on all infants. Forty two (93\%) of the respondents considered that the TEOAE screen was worth while with three being uncertain. Nevertheless, $39(87 \%)$ wanted to continue with the IDT with only one not wanting to and the remaining five being uncertain. Following circulation of the reported comparative evaluation the health visitors were asked whether they still felt the IDT should continue unchanged, or whether it should be dropped or continued as a selective screen. Such a selective IDT would be restricted to those at risk of deafness, those moving into the district, those who failed to complete the neonatal screen, and to those where professional or parental concern exists. Of 39 respondents, only eight $(20.5 \%)$ now considered that the IDT should continue unchanged; the remainder chose a selective screen later in infancy. Community paediatricians responded in the same way. The model of selective neonatal screening followed in the second half of infancy by a universal IDT will thus have been reversed within the district. Because the neonatal screen is $25 \%$ less expensive to implement than the distraction test, this reversal of usual practice permits identification and early habilitation without revenue consequences for the district.

1 Watkin, PM. Neonatal otoacoustic emission screening and the identification of deafness. Arch Dis Child 1996;74:F16F25.

2 Markides A. Age of fitting of hearing aids and speech intelligibility. Br $\mathcal{f}$ Audiol 1986;20:165-8.

3 Ramkalawan T, Davis AC. The effects of hearing loss and age of intervention on some language metrics in a population of young hearing impaired children. $B r \mathcal{F}$ Audiol 1992;26:97-107.

4 Robinshaw HM. Early intervention for hearing impairment: differences in the timing of communicative and linguistic development. Br f Audiol 1995;29:315-34. 
5 Bamford J. Clinical outcomes. In: Occasional papers in the field of early identification of hearing impairment in children. field of early identification

6 The National Deaf Children's Society. Quality standards in paediatric audiology. Guidelines for the early identification of hearing impairment.

7 Watkin PM, Baldwin M, Laoide S. Parental suspicion and the identification of hearing impairment. Arch Dis Child 1990;65:846-50.

8 Watkin PM. The age of identification of childhood deafness - improvements since the 1970s. Public Health 1991;105:303-12.

9 Ewing IR, Ewing AWG. The ascertainment of deafness in infancy and early childhood. F Laryngol Otol 1944;59:30938.

10 Hall DMB, ed. Health for all children. Report of the third join working party onchild health surveillance. Third edn. Oxford: working party onchild health survell

11 National Institutes of Health. Early identification of hearing impairment in infants and young children. NIH Consensus Statement

12 Watkin PM, Baldwin M, McEnery G. Neonatal at risk screening and the identification of deafness. Arch Dis Child 1991;66:1130-5.

$13 \mathrm{Kemp} D$, Ryan S. The use of transient evoked otoacoustic emissions in neonatal hearing screening programs. Semin Hear 1993;14:30-44.

14 British Society of Audiology Recommendations. Descriptors for pure tone audiograms. Br $\mathcal{F}$ Audiol 1988;22:28795.

15 Joint Committee on Infant Hearing. Position Statement 1982. Pediatrics 1982;70:496-7.

16 Haggard $M$. Hearing screening in children - state of the art(s). Arch Dis Child 1990;65:1193-8.
17 Sancho J, Hughes E, Davis A, Haggard M. Epidemiological basis for screeninghearing. In: McCormick B, ed. Paediatric audiology, 0-5 years. London:Taylor and Francis, ric audiolog

18 Kennedy CR, Kimm L, Dees DC, Evans PIP, Hunter M, Lenton $\mathrm{S}$, et al. Otoacoustic emissions and auditory brainstem responses in the newborn. Arch Dis Child 1991;66:1124-9.

19 Thompson M, Weber BA. Responses of infants and young children to behaviour observation audiometry. $\mathcal{F}$ Speech Hearing Dis 1974;39:140-7.

20 Wharrad H J. Neonatal hearing screening tests. In: McCormick B, ed. Paediatric audiology, 0-5 years. London:Taylor and Francis, 1988:69-95.

21 Tucker SM, Bhattacharya J. Screening hearing impairment in the newborn using the auditory response cradle. Arch Dis Child 1992;67:911-19.

22 Webb HD, Stevens JC. Auditory screening in high risk neonates; selection of a test protocol. Clin Phys Meas neonates; selection
1991;12:75-86.

23 Stuart A, Yang EY, Green WB. Neonatal auditory brainstem response thresholds to air and bone conducted clicks; 0 to 96 hours post partum. Fournal of the American Academy of Audiology 1994;5:163-72.

24 Sutton GJ, Gleadle P, Rowe SJ. Tympanometry and otoacoustic emissions in acohort of special care neonates. Br F Audiol 1996;30:9-17.

25 Gerber SE. Review of high risk register for congenital or early onset deafness. Br $\mathscr{Y}$ Audiol 1990;24:347-56.

26 McCormick B, Wood S, Cope Y, Spavins FM. Analysis of records from an open access audiology service. $\mathrm{Br} \mathcal{F}$ Audiol records from an ope.

27 Howie VM. Natural history of otitis media. Ann Otol Rhinol Laryngol 1975; 84 (Suppl 19):67-72. 